\title{
Serum Glial Cell Line-derived Neurotrophic Factor (sGDNF) is a Novel Biomarker in Predicting Cirrhosis in Patients With Chronic Hepatitis B
}

Cheng Liu ( $\nabla$ liucheng0082010@163.com )

Shanghai University of Traditional Chinese Medicine https://orcid.org/0000-0002-8741-6169

Guangyue Yang

SHUTCM: Shanghai University of Traditional Chinese Medicine

Liping Zhuang

Fudan University

Tiantian Sun

SHUTCM: Shanghai University of Traditional Chinese Medicine

YeeHui Yeo

Cedars-Sinai Medical Center

Le Tao

SHUTCM: Shanghai University of Traditional Chinese Medicine

Wei Zhang

SHUTCM: Shanghai University of Traditional Chinese Medicine

Wenting Ma

SHUTCM: Shanghai University of Traditional Chinese Medicine

Liu Wu

SHUTCM: Shanghai University of Traditional Chinese Medicine

Zongguo Yang

Fudan University

\section{Yanqin Yang}

SHUTCM: Shanghai University of Traditional Chinese Medicine

Dongying Xue

SHUTCM: Shanghai University of Traditional Chinese Medicine

Jie Zhang

SHUTCM: Shanghai University of Traditional Chinese Medicine

Rilu Feng

Heidelberg University

Ebert Matthias

Heidelberg University

Steven Dooley 
Heidelberg University

\section{Ekihiro Seki}

Cedars-Sinai Medical Center

\section{Ping Liu}

SHUTCM: Shanghai University of Traditional Chinese Medicine

\section{Research Article}

Keywords: glial cell line-derived neurotrophic factor (GDNF), cirrhosis, diagnostic accuracy, non-invasive biomarker

Posted Date: July 26th, 2021

DOl: https://doi.org/10.21203/rs.3.rs-740620/v1

License: (c) (i) This work is licensed under a Creative Commons Attribution 4.0 International License. Read Full License 
Title:

Serum glial cell line-derived neurotrophic factor (sGDNF) is a novel biomarker in predicting cirrhosis in patients with chronic hepatitis $\mathbf{B}$

Short running title: sGDNF is a biomarker for cirrhosis

Guangyue Yang ${ }^{1,2^{*}}$, Liping Zhuang ${ }^{3,4^{*}}$, Tiantian Sun ${ }^{1,2^{*}}$, YeeHui $\mathrm{Yeo}^{5}$, Le Tao ${ }^{1,2}$, Wei Zhang $^{1,2}$, Wenting $\mathrm{Ma}^{1,2}$, Liu Wu ${ }^{1,2}$, Zongguo Yang ${ }^{6}$, Yanqin Yang ${ }^{7}$, Dongying Xue ${ }^{1}$, Jie Zhang $^{1}$, Rilu Feng ${ }^{8}$, Ebert Matthias $^{8}$, Steven Dooley ${ }^{8}$, Ekihiro Seki ${ }^{9}$, Ping Liu ${ }^{10}$, Cheng $\mathrm{Liu}^{1,2}$

${ }^{1}$ Laboratory of Liver Disease, Department of Infectious Disease,

${ }^{2}$ Experimental Center, Putuo Hospital, Shanghai University of Traditional Chinese Medicine, Shanghai, 200062, China.

${ }^{3}$ Department of Integrative Oncology, Fudan University Shanghai Cancer Center; ${ }^{4}$ Department of Oncology, Shanghai Medical College, Fudan University; Shanghai, 200032, China.

${ }^{5}$ Division of General Internal Medicine, Department of Medicine, Cedars-Sinai Medical Center, Los Angeles, CA 90048, USA.

${ }^{6}$ Department of Integrative Medicine, Shanghai Public Health Clinical Center, Fudan University.

${ }^{7}$ Department of Pathology, Putuo Hospital, Shanghai University of Traditional Chinese 
Medicine, Shanghai, 200062, China.

${ }^{8}$ Department of Medicine II, Medical Faculty Mannheim, Heidelberg University, Mannheim, Germany.

${ }^{9}$ Division of Digestive and Liver Diseases, Department of Medicine, Cedars-Sinai Medical Center, Los Angeles, CA 90048, USA.

${ }^{10}$ Institute of Liver Diseases, Shuguang Hospital Affiliated to Shanghai University of Traditional Chinese Medicine, 528 Zhangheng Road, Pudong New District, Shanghai, 201203, China.

\section{\#these authors share equal work}

\section{Address for correspondence:}

Cheng Liu, Laboratory of Liver Disease, Department of Infectious Disease, Putuo Hospital, Shanghai University of Traditional Chinese Medicine. Email:liucheng0082010@163.com;

Ping Liu, Institute of Liver Diseases, Shuguang Hospital Affiliated to Shanghai University of Traditional Chinese Medicine. E-mail: liuliver@vip.sina.com;

Ekihiro Seki, Division of Digestive and Liver Diseases, Department of Medicine, Cedars-Sinai Medical Center. E-mail: Ekihiro.Seki@cshs.org 
Steven Dooley, Department of Medicine II, Medical Faculty Mannheim, Heidelberg University, Mannheim, Germany. Steven.Dooley@medma.uni-heidelberg.de

Word number: 2800

\begin{abstract}
Abbreviations: GDNF, glial cell line-derived neurotrophic factor; CHB, chronic hepatitis B; ELISA, enzyme-linked immunosorbent assay; AUROC, area under receiver operating characteristics curve; CI, confidence interval; AST, aspartate aminotransferase; FIB-4, fibrosis index based on four factors; AFP, alpha fetoprotein; Alb, albumin; ALP, alkaline phosphatase; ALT, alanine aminotransferase; ANOVA, analysis of variance; APRI, aspartate aminotransferase-to-platelet ratio index; OR, odds ratio; $\gamma$-GT, gamma-glutamyl transferase; HBV, hepatitis B virus; INR, international normalized ratio; IQR, interquartile range; PCR, polymerase chain reaction.
\end{abstract}

\title{
Conflict of interest
}

The authors have declared that no competing interest exists

\section{Financial support and Acknowledgment}

This work was mainly supported by The National Natural Science Foundation of China (no. 81673788,81873136 (to C.L), no. 81530101 (to P.L), no. 81803232 (to W.M), no. 81803898 (to L.T) and no. 82004106 (to L.W)). National Institutes of Health Grant (no. R01DK085252, R01AA027036 (to E.S)). Federal Ministry of Education and Research 
grant 'LiSyM' (to S.D). Shanghai Natural Science Foundation(no. 20ZR1450300 (to L.T)).

\section{Author contributions}

Yang GY, Zhuang LP, Sun TT, Ma WT, Tao L and Wu L carried out the experiments. Yeo YH, Liu C, Seki E, and Liu P conceived and designed the experiments. Yang ZG, Yang YQ, Xue DY, and Zhang J managed and studied human liver biopsy specimen. Dooley S, Feng R, and Matthias E performed data analysis. Liu C, Yeo YH, and Seki E wrote the manuscript, which was read, edited, and approved by all authors.

\section{Ethics approval and consent to participate}

Human samples and study protocol were approved by the Clinical Ethics Committee of Putuo Hospital, Shanghai University of Traditional Chinese Medicine and Shanghai Public Health Clinical Center, Fudan University. The study conforms with the provisions of the Declaration of Helsinki(as revised in Edinburgh 2000).

\section{Data Availability Statement}

The datasets used and analyzed during the current study are available from the corresponding author on reasonable request. 


\section{Abstract}

We assessed the potential of glial cell line-derived neurotrophic factor (GDNF) as a useful biomarker to predict cirrhosis in chronic hepatitis $\mathrm{B}(\mathrm{CHB})$ patients.A total of 735 patients from two medical centers ( $385 \mathrm{CHB}$ patients and 350 healthy controls) were included to determine the association of serum and tissue GDNF levels with biopsy-proven cirrhosis. The diagnostic accuracy of serum GDNF (sGDNF) was estimated and compared with other indices of cirrhosis. We showed significantly higher levels of sGDNF in CHB patients with fibrosis $(28.4 \mathrm{pg} / \mathrm{ml}$ vs. $11.6 \mathrm{pg} / \mathrm{ml}$ in patients without) and patients with cirrhosis (33.8 pg/ml vs. $23.5 \mathrm{pg} / \mathrm{ml}$ in patients without). The areas under receiver operating curve (AUROCs) of sGDNF were 0.83 ( $95 \%$ confidence interval (CI): $0.80-0.87)$ for predicting liver fibrosis and 0.84 (95\% CI: $0.79-0.89)$ for cirrhosis. Findings from serum protein level and hepatic mRNA expression were consistent. Using the best cutoff to predict cirrhosis, we categorized the patients into sGDNF-high and sGDNF-low groups. The sGDNF-high group had significantly larger Masson's trichrome and reticulin staining-positive area, higher Scheuer score and METAVIR fibrosis stage (all $p<0.001$ ) but not steatosis. On multivariable regression, sGDNF was independently associated with cirrhosis with an odds ratio at 6.98 (95\% CI: 1.10-17.94). Finally, we demonstrated that sGDNF outperformed AST to platelet ratio index, FIB-4, fibroscore, forn index, and fibrometer in differentiating F4 vs. F3. Using serum, tissue mRNA, and biopsy data, our study revealed a significant potential of sGDNF as a novel non-invasive biomarker for cirrhosis in CHB patients. 


\section{Keywords}

glial cell line-derived neurotrophic factor (GDNF), cirrhosis, diagnostic accuracy, noninvasive biomarker.

\section{Introduction}

Cirrhosis is the leading risk factor of hepatocellular carcinoma and is associated with premature death [1]. Given the high risk of complications from hepatic decompensation, cirrhosis leads to substantial health burden [2]. Early detection and treatment of cirrhosis may reduce the risk of disease progression and development of complications. In hepatitis B virus-infected patients, indefinite antiviral treatment is recommended if patients develop cirrhosis [3]. Therefore, early detection of cirrhosis in patients with chronic hepatitis B infection (CHB) is important in informing medical decisions.

Although percutaneous liver biopsy and histological assessment remained the gold standard for diagnosing liver fibrosis [4], the invasiveness limits its wide application [5]. Additionally, the accuracy of hepatic fibrosis assessment is limited by both sampling error and inter-observer variability between pathologists. Non-invasive techniques (eg. serum biomarkers and imaging) are widely performed in countries where these techniques are available and approved [6-8]. Liver stiffness with transient elastography (TE) and magnetic resonance elastography (MRE) are well-validated methods for the assessment of liver fibrosis and cirrhosis $[6,9,10]$. However, these methods are costly and limited to certain liver centers. Moreover, MRE is challenging 
to perform in some cases, such as for patients with severe iron overload, claustrophobia, or other MR contraindications. Direct serum biomarkers and indirect serum composite scores, such as aspartate aminotransferase to platelet ratio index (APRI), FIB-4 index, and Fibrotest are widely used for noninvasive hepatic fibrosis assessment; they are more affordable and can be applied in most clinical settings [11-13]. However, their diagnostic accuracies are limited. Therefore, unmet medical needs for novel biomarkers with better diagnostic performance is significant.

GDNF is a glycosylated, disulfide-bonded homodimer that is a distantly related member of the TGF- $\beta$ superfamily [14]. Clinical studies have found that the GDNF level is increased in the parietal cortex and plasma of recurrent major depressive disorder patients [15]. Additionally, GDNF is increased by several folds following exposure to cytotoxic agents, including radiation [16]. Additionally, GDNF levels are increased in some cancer cell types [17].

Recently, we reported that GDNF promotes hepatic stellate cell activation and liver fibrosis via ALK5/Smad signaling in the preclinical mouse models of liver fibrosis [18]. We also found that hepatic GDNF levels were upregulated in human liver fibrosis [18]. However, the clinical use of GDNF in liver disease remains unclear. In the present study, we assessed GDNF along with biochemical and histological parameters of liver disease in CHB patients. We determined the diagnostic accuracy of serum GDNF in liver fibrosis and cirrhosis and compared that with other known markers. 


\section{Patients and Methods}

Human samples and study protocol were approved by the Clinical Ethics Committee of Putuo Hospital, Shanghai University of Traditional Chinese Medicine and Shanghai Public Health Clinical Center, Fudan University. The study conforms with the provisions of the Declaration of Helsinki.

The CHB diagnosis was confirmed by the presence of hepatitis B surface antigen for more than 6 months. All patients underwent clinical, biochemical, virological examination, and liver biopsy on the same day. Patients with renal and/or hepatic failure, acute coronary syndromes, valvular heart diseases, autoimmune thyroid diseases, or systematic inflammatory diseases were excluded from our study. Additionally, patients with prior anti-viral therapy were excluded.

A total of $385 \mathrm{CHB}$ patients with serum and biopsy samples and among them, 293 with frozen tissue-derived GDNF mRNA results were included. Serum samples were also obtained from 350 healthy controls who underwent physical examination from December 2017 to July 2019 (Supplemental Figure 1). Serum samples and liver biopsy were collected on the same day from 344 CHB patients at the Putuo Hospital from June 2011 to July 2019. Among them, liver biopsy frozen tissue was procured from 252 patients to determine the liver GDNF mRNA expression. We also collected liver biopsy frozen tissue samples from 41 patients who visited the Shanghai Public Health Clinical Center from November 2013 to March 2016. 


\section{Histological liver fibrosis staging}

Liver biopsy specimens were obtained using $16 \mathrm{G}$ x $20 \mathrm{~cm}$ disposable needles (Cat no. MACП, Mantova, Italy). The biopsy specimens were then fixed in $4 \%$ formalin and embedded in paraffin. Adequate specimens were required to be at least $15 \mathrm{~mm}$ in length, and the sections ( $3 \mathrm{~mm}$ thick) were stained with hematoxylin and eosin (HE), reticulin, and Masson's trichrome $[19,20]$. The stage of liver fibrosis was scored based on the examination of HE, Masson's trichrome, and reticulin staining by three independent pathologists who were blind to the clinical characteristics of the study subjects at the Putuo Hospital or Shanghai Public Health Clinical Center Department of Pathology. Fibrosis stages were defined based on Scheuer criteria and METAVIR scoring system [20]. According to the Scheuer scoring system, the severity of liver injury was categorized into G0, G1, G2, G3, and G4, with G1 defined as portal inflammation; G2 as mild piecemeal necrosis; G3 as moderate piecemeal necrosis; G4 as severe piecemeal necrosis and bridging necrosis. According to METAVIR scoring system, the severity of liver fibrosis was categorized into F0, F1, F2, F3, and F4. F0 was defined as no fibrosis; F1 as portal fibrosis without septa; F2 as septal fibrosis (portal-portal); F3 as septal fibrosis (portal-central), and F4 as cirrhosis.

The images of Masson's trichrome and reticulin staining were captured using an BX43 Olympus microscpoe (Olympus Tokoyo, Japan) and processed using DP73 version software. To quantify Masson's trichrome and reticulin staining, images of five or six 
randomly chosen fields of each section were taken. The collagen values are expressed as the percentage of the area of the section occupied by Masson's trichrome and reticulin staining using Image-Pro-plus (IPP) software (Media Cybernetics, MA, USA)

\section{Biochemical analyses.}

The serum were collected on the same day as biopsy. The following parameters were assessed: alanine transaminase (ALT), aspartate transaminase (AST), alkaline phosphatase (ALP), gamma glutamyltransferase (GGT), total bilirubin, prothrombin time, international normalized ratio (INR), fasting glucose, albumin, hemoglobin, platelets, leukocytes, triglycerides, haptoglobin, and cholesterol and its components. All samples, including those from the Shanghai Public Health Clinical Center, were determined using standardized assays and methods from the Department of Clinical Laboratory, Putuo Hospital.

Hyaluronic acid (HA), type IV collagen (CIV), laminin (LN), and type III procollagen (PCIII) were assessed using radiometric assays at the Department of Nuclear Medicine, Putuo Hospital. a2-macroglobulin was determined by Dian Diagnostics Co., Ltd (Shanghai, China).

\section{Definition of indices for liver cirrhosis}

APRI: $(\mathrm{AST}(\mathrm{U} / \mathrm{L}) /$ upper normal limit $) \times 100 /$ platelets $\left(10^{9} / \mathrm{L}\right)[21]$.

FIB-4 index: age $($ years $) \times$ AST $(\mathrm{U} / \mathrm{L}) /\left(\operatorname{PLT}\left(10^{9} / \mathrm{L}\right)\right) \times\left(\mathrm{ALT}(\mathrm{U} / \mathrm{L})^{1 / 2}\right)[22]$. 
Fibrometer: -0.007 PLT (G/L)-0.049 PI (\%) + 0.012AST (U/L) + $0.005 \alpha 2 \mathrm{M}(\mathrm{mg} / \mathrm{dL})$ $+0.021 \mathrm{HA}(\mu \mathrm{g} / \mathrm{L})-0.270$ urea $(\mathrm{mmol} / \mathrm{L})+0.027$ age $(\mathrm{yr})+3.718[23]$

Forn index: $7.811-3.131 \times \ln \left(\mathrm{PLT}\left(10^{9} / \mathrm{L}\right)\right)+0.781 \times \ln (\mathrm{GGT}(\mathrm{U} / \mathrm{L}))+3.467 \times$ $\ln ($ age $)-0.014 \times($ cholesterol $(\mathrm{mg} / \mathrm{dl})[23]$.

Hepascore: $\frac{y}{y+1}$ $\mathrm{y}=\exp [4.185818-(0.0249 \times$ age $)+(0.7464 \times \operatorname{sex})+(1.0039 \times \alpha 2 \mathrm{Mg} / \mathrm{L})+$ $(0.0302 \times \mathrm{HA} \mu \mathrm{g} / \mathrm{L})+(0.0691 \times$ bilirubin $\mu \mathrm{mol} / \mathrm{L})-(0.0012 \times \mathrm{GGT} \mathrm{U} / \mathrm{L})]$, male $=1$, female $=0$ [24].

Fibrotest: $4.467 \times \log (\alpha 2 \mathrm{M}(\mathrm{mg} / \mathrm{dL}))-1.357 \times \log \operatorname{Hap}(\mathrm{g} / \mathrm{L})+1.017 \log \mathrm{GGT}(\mathrm{U} / \mathrm{L})$ $+0.0281 \times$ age $+1.737 \times \log$ TBil $(\mu \mathrm{mol} / \mathrm{L})-1.184 \times$ apoA-I $(\mathrm{g} / \mathrm{L})+0.301 \times$ (sex)-5.540, male $=1$, female $=0[12,13]$.

\section{Statistical Methods}

The PASW Statistics software version 23.0 from SPSS Inc. (Chicago, IL, USA) was used for all analyses. Data were expressed as mean \pm standard deviation or median (interquartile range) as appropriate. One-way analysis of variance (ANOVA) was used for comparison of multiple groups, and student's t test was applied to examine the mean differences in normally distributed continuous variables between groups.

The correlation of clinical, biological, and histological factors with cirrhosis was analyzed using stepwise forward multivariable logistic regression. Variables that showed a $p<0.05$ on univariable logistic regression were selected for multivariable regression. 
To determine the diagnostic accuracy of GDNF and other indices, area under receiver operating characteristic, sensitivity, specificity, positive predictive value (PPV), and negative predictive value (NPV) were calculated. The optimal cut-off of GDNF and all indices were determined using Youden index. DeLong test was used to compare the AUROCs of GDNF to several commonly used indices in predicting cirrhosis $[10,25]$ using Medcalc software version 15.8 (Ostend, Belgium).

For other materials, please see Supplemental materials. 


\section{Results}

\section{Patient characteristics}

As shown in Table 1A, patients with fibrosis/cirrhosis (METAVIR stage F1-F4, N=318) had higher serum GDNF (sGDNF) levels $(28.4 \mathrm{pg} / \mathrm{ml}$ [IQR: 26.2, 31.6]) than patients without fibrosis $(11.6 \mathrm{pg} / \mathrm{ml}$ [IQR: $7.2,21.1])$ (healthy controls and METAVIR stage $\mathrm{F} 0, \mathrm{~N}=376)(p<0.001)$. Meanwhile, cirrhotic patients (METAVIR stage $\mathrm{F} 4, \mathrm{~N}=33)$ had high sGDNF levels $(33.8 \mathrm{pg} / \mathrm{ml}$ [IQR: 29.3, 39.4]) as compared with non-cirrhotic patients (healthy controls and METAVIR stage F0-F3, N=661) (23.5 pg/ml [IQR: 10.9, 29.5]) $(p<0.001)$ (Table 1B). Other patient characteristics are also summarized in Table

\section{$1 \mathrm{~A}$ and $\mathrm{B}$.}

\section{Evidence for sGDNF-based fibrosis and cirrhosis prediction}

To investigate the diagnostic accuracy of sGDNF levels for diagnosing liver fibrosis and cirrhosis, we calculated the areas under receiver operating curve (AUROC) values of sGDNF (Figure 1). The AUROCs of sGDNF for diagnosing fibrosis and cirrhosis were $0.83(0.80-0.87)$ and $0.84(0.79-0.89)$, respectively. Using Youden's index, we determined that the best cutoff value of sGDNF in differentiating cirrhotics and noncirrhotics was $28.74 \mathrm{pg} / \mathrm{ml}$.

\section{Clinical characteristics and sGDNF}

We further categorized the $344 \mathrm{HBV}$ patients into sGDNF-low $(<28.74, \mathrm{~N}=192)$ and sGDNF-high $(\geq 28.74, \mathrm{~N}=152)$ groups (Supplemental Table 1). sGDNF-high group 
demonstrated lower serum Alb level $(p=0.008)$ as well as higher PCIII $(p=0.012)$ and LN ( $p=0.047)$ values than sGDNF-low group. There was no significant difference in demographic characteristics, viral load, liver enzymes, and coagulation factors.

\section{Serum and mRNA levels of GDNF in different histopathological categories}

By examining biopsy samples, we showed that sGDNF-high and sGDNF-low groups did not have significant differences in steatosis $(p=0.556)$ (Table 2). Consistently, there were no mean differences in sGDNF levels between subgroups of steatosis (Figure 2). A slight difference in sGDNF level was found when comparing among G0-1, G2, and G3-4 inflammation stages. Regarding biopsy-proven fibrosis, sGDNF-high group had higher fibrosis stages $(p<0.001)$, larger reticulin- $(p=0.023)$ and Masson's trichromepositive area $(p=0.004)$ than sGNDF-low group (Table 2). Consistently, there was a significantly increasing trend in mean sGDNF levels across the fibrosis stage (Figure 2).

As shown in Supplemental Figure 2, the hepatic mRNA expression of GDNF was in accordance with serum and histopathological examination. Patients with higher G stage and fibrosis stage had significantly higher hepatic mGDNF levels $(p<0.001)$ and there was no significant difference when patients were categorized by steatosis and necrosis stages.

\section{sGDNF as an independent factor of cirrhosis}


We conducted a multivariable logistic regression analysis to determine the factors that were associated with cirrhosis (Table 3). After adjusting for a variety of clinical characteristics, cirrhosis was significantly associated with albumin (adjusted odds ratio [aOR] 0.85; 95\% CI, 0.74-0.97, $p=0.019$ ), G stage (aOR 5.55; 95\% CI, 1.56-19.67, $p=0.008$ ), and sGDNF (aOR 6.98; 95\% CI, 1.10-17.94, $p=0.036$ ).

sGDNF was superior to APRI, FIB-4, Fibrometer, Forn index, and Hepascore in predicting cirrhosis in CHB patients.

We compared the diagnostic performance of sGDNF with those of APRI, FIB-4, fibrometer, hepascore, forn index, and fibrotest in CHB patients (Table 4). sGDNF (AUROC 0.74 [95\% CI, 0.65-0.83]) had a significantly higher AUROC than APRI (0.55 [95\% CI, 0.44-0.65]; $p=0.0124)$, FIB-4 (0.55 [95\% CI, 0.46-0.65]; $p=0.0181)$, fibrometer $(0.53 ; 95 \% \mathrm{CI}, 0.43-0.64 ; p=0.0133)$, hepascore $(0.55 ; 95 \% \mathrm{CI}, 0.45-0.65$; $p=0.0211)$, and forn index $(0.57$ [95\% CI, 0.47-0.67]; $p=0.0368)$, but not fibrotest $(0.61$ [95\% CI, 0.47-0.67]; $p=0.0802$ ) to diagnose F4 vs F3. No significant difference was noted when comparing the diagnostic accuracy between sGDNF and these indices to diagnose cirrhosis vs. no cirrhosis (F4 vs F0-3). 


\section{Discussion}

In this study, we showed that patients with biopsy-proven cirrhosis and fibrosis demonstrated higher serum protein and tissue mRNA levels of GDNF. A significantly dose-dependent association of sGDNF level and METAVIR fibrosis stage was observed. sGDNF was associated with significantly higher odds of cirrhosis after adjusting for clinical characteristics. Furthermore, when comparing between fibrosis stage F3 and F4, sGDNF outperformed most indices for liver cirrhosis including APRI, FIB-4, Fibrometer, Forn index, and Hepascore.

We recently reported that GDNF is the functional promoter of hepatic stellate cell activation and liver fibrosis mediated through ALK5/Smad signaling. We further suggest that GDNF inhibition could be a therapeutic strategy for patients with liver fibrosis[18]. In this study, we observed consistent clinical pictures in CHB patients. We found that sGDNF-high patients have a higher Scheuer score (represented as G stages), larger Masson's trichrome and reticulin staining positive areas, and higher METAVIR stages. Masson's trichrome staining represents the accumulation of collagen fiber and thus serves as a gold-standard method to diagnose liver fibrosis histologically. Reticulin staining is also a useful tool to stain type III collagen fiber for diagnosing fibrosis severity. Therefore, the results indicated that sGDNF is correlated with inflammation and extracellular matrix production and deposition, which were in line with our previous preclinical studies. Furthermore, the results from sGDNF levels and tissue GDNF mRNA levels were consistent, which enhanced the validation of the findings. 
Besides, we also showed that both by serum protein level and tissue mRNA expression in the liver, GDNF was specifically correlated with liver fibrosis but not with the pattern of necrosis and the existence of steatosis. This exemplified the mechanism of GDNFmediated liver fibrosis and implied that sGDNF might serve as a powerful non-invasive biomarker for diagnosing fibrosis and cirrhosis. Comparably, on the multivariable logistic regression analysis with an extensive adjustment for clinical confounders, including liver function panels, fibrogenic factors, and blood counts, sGDNF levels were significantly associated with cirrhosis. Other markers, such as platelet counts, AST, ALT, and total bilirubin levels only showed significances on a univariable but not a multivariable regression.

Multiple studies have used serum markers to predict liver fibrosis. The fibrosis parameters commonly used are related to hepatocyte damage (ALT, AST), macrophages [26], microbiota [27], and hepatic stellate cell activation [28, 29], these markers could predict advanced fibrosis (F3-4 vs F0-2) or cirrhosis (F4 vs F0-3). However, the markers that can predict F4 vs F3 are uncommon. Given that patients with F3 had a significantly lower risk of hepatic decompensation, hepatocellular carcinoma, overall mortality and higher transplant free survival rate, the clinical relevance to diagnose between F4 vs F3 is crucially important $[30,31]$. Our results show that sGDNF level can be a first-class predictor for distinguishing F4 cirrhosis from F3 fibrosis. Moreover, comparing sGDNF levels with ARPI, FIB-4, fibrotest, fibrometer, hepascore, and forn index, we 
found that sGDNF is significantly superior in predicting fibrosis stage F4 vs F3.

The first strength of this study is that we included patients with biopsy-proven fibrosis/cirrhosis to investigate the correlation between sGDNF levels and the severity of liver fibrosis. Secondly, the results were consistent between serum protein levels and liver tissue mRNA expression of GDNF. Thirdly, we were able to include a large sample size of patients with comprehensive clinical and histological data, which allowed us to minimize residual confounders on multivariable analysis. Fourthly, the pathologists who examined the liver samples were blind to the patients' information.

Our study still has some limitations. Firstly, we only included CHB patients and thus the results may not be able to be generalized to patients with other chronic liver diseases. However, in our previous preclinical study, we showed that GDNF level was associated with liver fibrosis developed in several different etiologies [18]. Studies that enroll cirrhotic patients of other primary liver diseases are needed to expand the diagnostic application of sGDNF. Secondly, to enhance the comparability, we only determined the differences between sGDNF levels and other serum marker-based indices. However, we showed that the AUROCs of sGDNF to diagnose cirrhosis (F4) and fibrosis (F1-3) were 0.84 and 0.83 , respectively. A previous meta-analysis demonstrated that the pooled AUROCs of acoustic radiation force impulse elastography for examining cirrhosis (F4) and evident fibrosis ( $\geq \mathrm{F} 2)$ were 0.93 and 0.85 , respectively [32]. Future studies that compare sGDNF with imaging modalities are required because imaging 
modalities, including MRE and FibroScan, are also useful and accurate in diagnosing liver fibrosis/cirrhosis[33], but they are not as available as serum marker tests in resource-limiting areas and the cost of the test may limit their widespread use.

\section{Conclusions}

In conclusion, we demonstrated that SGDNF is an accurate non-invasive biomarker for diagnosing cirrhosis in CHB patients. The accuracy in differentiating F4 vs F3 was superior to currently available indices, such as APRI, FIB-4 index, Fibrometer, Forn index, and Hepascore. Validation of its performance in cirrhotic patients of other primary liver diseases is also required. 


\section{References}

1. Thiele M, Detlefsen S, Sevelsted Moller L, et al., Transient and 2-Dimensional Shear-Wave Elastography Provide Comparable Assessment of Alcoholic Liver Fibrosis and Cirrhosis. Gastroenterology.2016;150(1):123-133. 10. 1053/j. gastro. 2015. 09. 040.

2. Zou B, Yeo Y H, Jeong D, Park H, Nguyen M H, A Nationwide Study of Inpatient Admissions, Mortality, and Costs for Patients with Cirrhosis from 2005 to 2015 in the USA. Digestive Diseases and Sciences.2020;65(375 - 382)

3. Terrault N A, Lok A S F, McMahon B J, et al., Update on prevention, diagnosis, and treatment of chronic hepatitis B: AASLD 2018 hepatitis B guidance. Hepatology. 2018;67 (4) :1560-1599. 10. 1002/hep. 29800.

4. Mani H, Kleiner D E, Liver biopsy findings in chronic hepatitis B. Hepatology. 2009;49 (Supp1 5) (5 Supp1):S61-71. 10. 1002/hep. 22930.

5. Sandrin L, Fourquet B, Hasquenoph J M, et al., Transient elastography: a new noninvasive method for assessment of hepatic fibrosis. Ultrasound in Medicine \& Biology. 2003;29 (12) : 1705-1713. 10. 1016/S0301-5629 (03) 01071-8.

6. Poynard T, de Ledinghen V, Zarski J P, et al., Relative performances of FibroTest, Fibroscan, and biopsy for the assessment of the stage of liver fibrosis in patients with chronic hepatitis C: a step toward the truth in the absence of a gold standard. J Hepato1.2012;56 (3):541-8. 10. 1016/j. jhep. 2011. 08. 007.

7. Thiele M, Madsen B S, Hansen J F, et al., Accuracy of the Enhanced Liver Fibrosis Test vs FibroTest, Elastography, and Indirect Markers in Detection of Advanced Fibrosis in Patients With Alcoholic Liver Disease. Gastroenterology. 2018;154(5) :1369-1379. 10. 1053/j. gastro. 2018.01. 005.

8. Jeon M Y, Lee H W, Kim S U, et al., Subcirrhotic liver stiffness by FibroScan correlates with lower risk of hepatocellular carcinoma in patients with HBV-related cirrhosis. Hepatol Int.2017;11(3):268-276. 10. 1007/s12072-017-9789-y.

9. Imajo K, Kessoku T, Honda Y, et al., Magnetic Resonance Imaging More Accurately Classifies Steatosis and Fibrosis in Patients With Nonalcoholic Fatty Liver Disease Than Transient Elastography. Gastroenterology.2016;150 (3):626637. 10. 1053/j. gastro. 2015. 11. 048.

10. Park C C, Nguyen P, Hernandez C, et al., Magnetic Resonance Elastography vs Transient Elastography in Detection of Fibrosis and Noninvasive Measurement of Steatosis in Patients With Biopsy-Proven Nonalcoholic Fatty Liver Disease. Gastroenterology. 2017;152 (3):598-607. 10. 1053/j. gastro. 2016. 10. 026.

11. Kim W R, Berg T, Asselah T, et al., Evaluation of APRI and FIB-4 scoring systems for non-invasive assessment of hepatic fibrosis in chronic hepatitis B patients. J Hepato1. 2016;64(4) :773-80. 10. 1016/j. jhep. 2015. 11. 012.

12. Imbert-Bismut F, Ratziu V, Pieroni L, et al., Biochemical markers of liver fibrosis in patients with hepatitis $\mathrm{C}$ virus infection: a prospective study. The Lancet. 2001 ; 357 (9262) : 1069-1075. 10. 1016/s0140-6736 (00) 04258-6.

13. Naveau S, Gaude G, Asnacios A, et al., Diagnostic and prognostic values of noninvasive biomarkers of fibrosis in patients with alcoholic liver disease. Hepatology. 2009; 49 (1) :97-105. 10. 1002/hep. 22576. 
14. Lin L F, Doherty D H, Lile J D, Bektesh S, Collins F, GDNF: a glial cell linederived neurotrophic factor for midbrain dopaminergic neurons. Science. $1993 ; 260$ (5111) : 1130-2

15. Park Y M, Lee B H, Alterations in Serum BDNF and GDNF Levels after 12 Weeks of Antidepressant Treatment in Female Outpatients with Major Depressive Disorder. Psychiatry Investig. 2018;15(8):818-823. 10. 30773/pi. 2018. 03. 31.

16. Huber R M, Lucas J M, Gomez-Sarosi L A, et al., DNA damage induces GDNF secretion in the tumor microenvironment with paracrine effects promoting prostate cancer treatment resistance. Oncotarget. 2015;6(4):2134-47. 10. 18632/oncotarget. 3040.

17. Zhong Z, Gu H, Peng J, et al., GDNF secreted from adipose-derived stem cells stimulates VEGF-independent angiogenesis. Oncotarget.2016;7 (24):3682936841. 10. 18632/oncotarget. 9208.

18. Tao L, Ma W, Wu L, et al., Glial cell line-derived neurotrophic factor (GDNF) mediates hepatic stellate cell activation via ALK5/Smad signalling. Gut.2019;68 (12) :2214-2227. 10. 1136/gutjn1-2018-317872.

19. Desmet V J, Gerber M, Hoofnagle J H, Manns M, Scheuer P J, Classification of Chronic Hepatitis: Diagnosis, Grading and Staging. Hepatology. 1994;19(6):1315-1320

20. Goodman Z D, Grading and staging systems for inflammation and fibrosis in chronic liver diseases. J Hepato1. 2007 ;47 (4):598-607. 10. 1016/j. jhep. 2007. 07. 006.

21. Wai C T, Greenson J K, Fontana R J, et al., A simple noninvasive index can predict both significant fibrosis and cirrhosis in patients with chronic hepatitis C. Hepatology. $2003 ; 38$ (2) :518-26. 10. 1053/jhep. 2003. 50346.

22. Sterling R K, Lissen E, Clumeck N, et al., Development of a simple noninvasive index to predict significant fibrosis in patients with HIV/HCV coinfection. Hepatology. 2006 ; 43 (6) :1317-25. 10. 1002/hep. 21178.

23. Forns X, Ampurdanes S, Llovet J M, et al., Identification of chronic hepatitis $\mathrm{C}$ patients without hepatic fibrosis by a simple predictive model. Hepatology. 2002;36 (4 Pt 1) :986-92. 10. 1053/jhep. 2002. 36128.

24. Adams L A, Bulsara M, Rossi E, et al., Hepascore: an accurate validated predictor of liver fibrosis in chronic hepatitis C infection. Clin Chem.2005;51(10):186773. 10. 1373/clinchem. 2005. 048389.

25. DeLong E R, DeLong D M, Clarke-Pearson D L, Comparing the areas under two or more correlated receiver operating characteristic curves: a nonparametric approach. Biometrics. 1988;44 (3) :837-45

26. Kazankov K, Barrera F, Moller H J, et al., Soluble CD163, a macrophage activation marker, is independently associated with fibrosis in patients with chronic viral hepatitis B and C. Hepatology.2014;60(2):521-30. 10. 1002/hep. 27129.

27. Lelouvier B, Servant F, Paisse S, et al., Changes in blood microbiota profiles associated with liver fibrosis in obese patients: A pilot analysis. Hepatology. 2016;64 (6) :2015-2027. 10. 1002/hep. 28829.

28. Leroy V, Monier F, Bottari S, et al., Circulating matrix metalloproteinases 1, 2, 9 and their inhibitors TIMP-1 and TIMP-2 as serum markers of liver fibrosis in patients with chronic hepatitis C: comparison with PIIINP and hyaluronic acid. Am J Gastroenterol.2004;99(2) :271-9 
29. Cao Z, Li Z, Wang H, et al., Algorithm of Golgi protein 73 and liver stiffness accurately diagnoses significant fibrosis in chronic HBV infection. Liver Int. $2017 ; 37$ (11) :1612-1621. 10. 1111/1iv. 13536.

30. Xu F, Moorman A C, Tong X, et al., Al1-Cause Mortality and Progression Risks to Hepatic Decompensation and Hepatocellular Carcinoma in Patients Infected With Hepatitis C Virus. Clinical Infectious Diseases.2016;62(3):289297. 10. 1093/cid/civ860.

31. Lin W, Axley P, Mudumbi S, et al., Patients with stage 3 compared to stage 4 liver fibrosis have lower frequency of and longer time to liver disease complications. Plos One. 2018;13(5). 10. 1371/journal. pone. 0197117.

32. Bota S, Herkner H, Sporea I, et al., Meta-analysis: ARFI elastography versus transient elastography for the evaluation of liver fibrosis. Liver Int. 2013;33 (8) :1138-47. 10. 1111/1iv. 12240.

33. Bradley C R, Cox E F, Scott R A, et al., Multi-organ assessment of compensated cirrhosis patients using quantitative magnetic resonance imaging. J Hepato1. 2018;69 (5) :1015-1024. 10. 1016/j. jhep. 2018. 05. 037. 


\section{Figure Legends}

Figure 1 Receiver operating characteristics (ROC) analysis showing the predictive value of sGDNF for liver fibrosis in patients with $\mathrm{CHB}$.

Receiver operating characteristic curve for sGDNF predicting liver fibrosis (A) and cirrhosis (B). The estimates indicate area under the ROC curve.

Figure 2 sGDNF according to the histological grade and fibrosis stage

sGDNF levels according to the histological grade and fibrosis stage in 344 patients.

Liver histopathology of patients with G0, G1, G2, G3, and G4 according to the Scheuer scoring system. G1, portal inflammation; G2, mild piecemeal necrosis; G3, moderate piecemeal necrosis; G4, severe piecemeal necrosis and bridging necrosis. METAVIR scoring system. F0, no fibrosis; F1, Portal fibrosis without septa; F2, Septal fibrosis (portal-portal); F3, Septal fibrosis (portal-central); F4, Cirrhosis. 
Figures
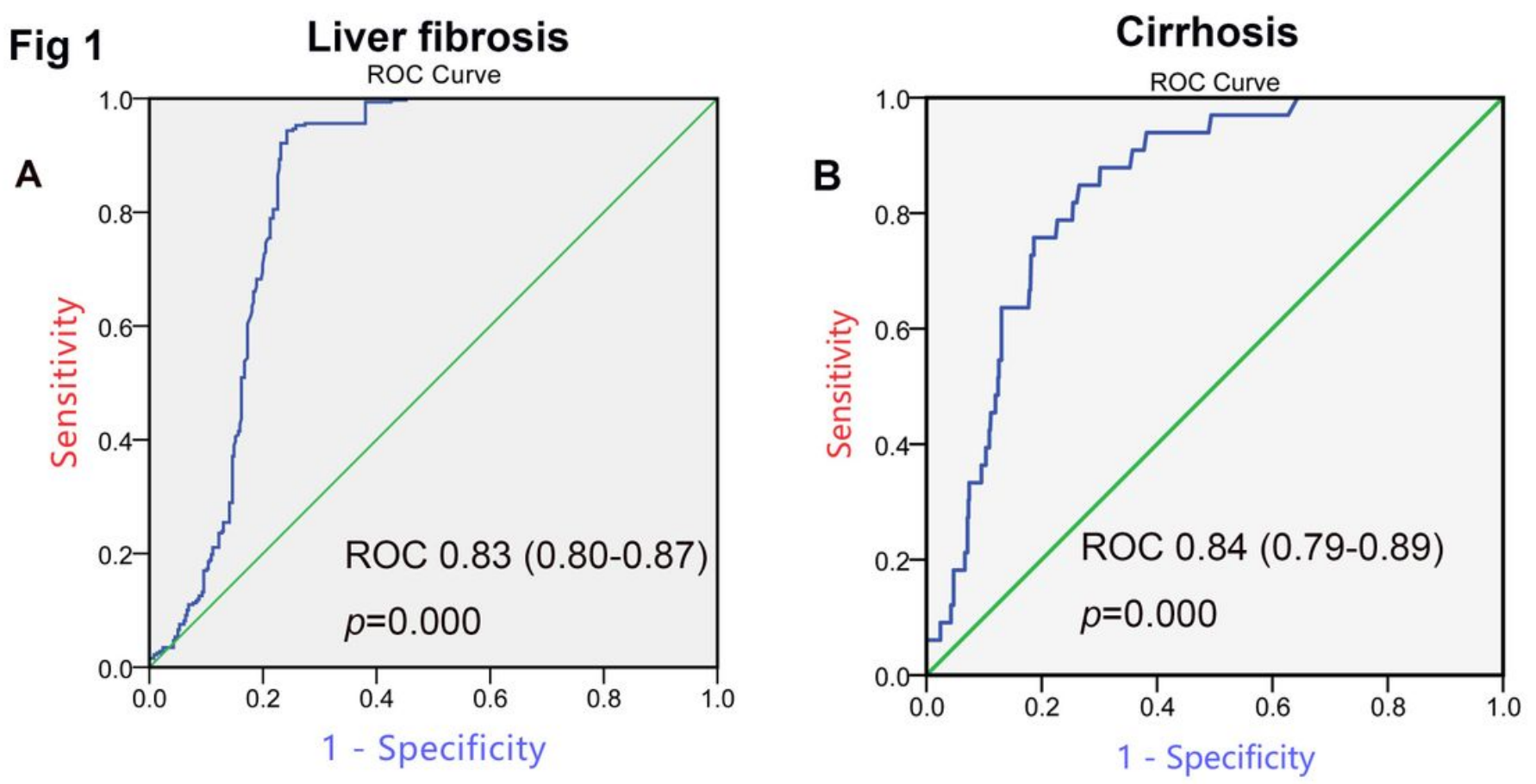

Figure 1

Receiver operating characteristics (ROC) analysis showing the predictive value of sGDNF for liver fibrosis in patients with CHB. Receiver operating characteristic curve for SGDNF predicting liver fibrosis $(A)$ and cirrhosis (B). The estimates indicate area under the ROC curve.

Fig 2

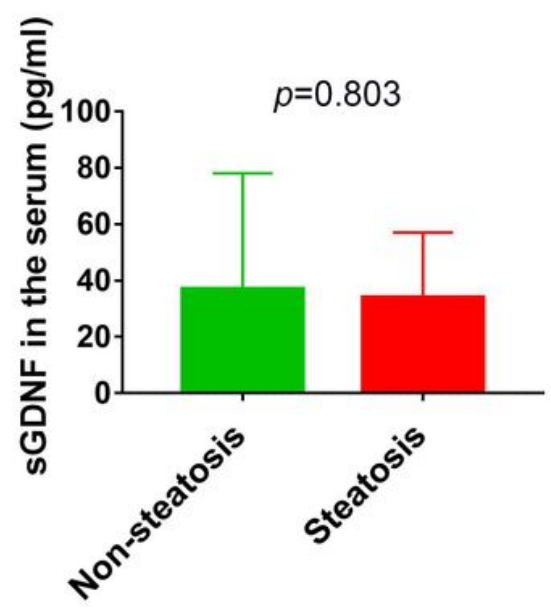

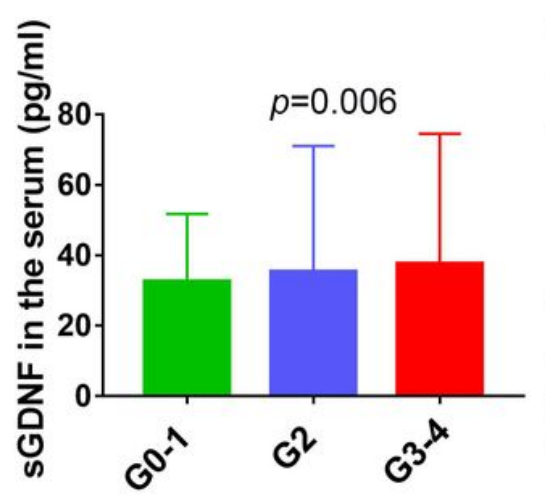

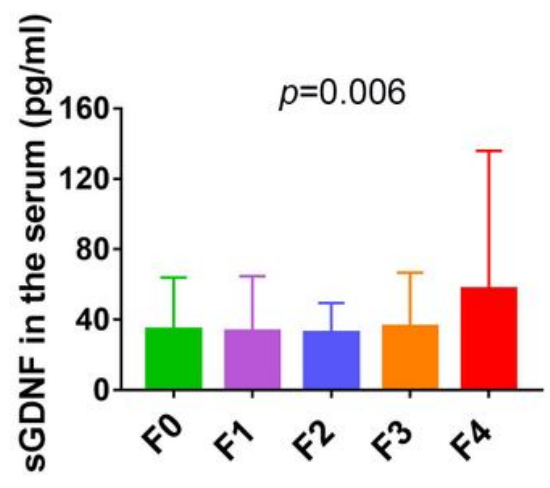

Figure 2 
sGDNF according to the histological grade and fibrosis stage sGDNF levels according to the histological grade and fibrosis stage in 344 patients. Liver histopathology of patients with G0, G1, G2, G3, and G4 according to the Scheuer scoring system. G1, portal inflammation; G2, mild piecemeal necrosis; G3, moderate piecemeal necrosis; $\mathrm{G} 4$, severe piecemeal necrosis and bridging necrosis. METAVIR scoring system. F0, no fibrosis; F1, Portal fibrosis without septa; F2, Septal fibrosis (portal-portal); F3, Septal fibrosis (portal-central); F4, Cirrhosis. 